dently (dcSSc: $P=3.20 E-2, O R=1.13 ; P A H: P=2.19 E-02, O R=1.32$ ). However, our data revealed a stronger effect size with the subset of SSc patients showing both clinical manifestations (dcSSc with $\mathrm{PAH}: \mathrm{P}=6.91 \mathrm{E}-3, \mathrm{OR}=2.05$ ).

Conclusions: We revisited the association of the MIF rs $755622^{*} \mathrm{C}$ allele with SSc and described a phenotype-specific association of this variant with the susceptibility to develop PAH in dSSc patients.

Disclosure of Interest: None declared

DOI: 10.1136/annrheumdis-2017-eular.1907

\section{AB0004 PSORIATIC ARTHRITIS. GENES INVOLVED IN THE MESTIZO POPULATION}

L.A. Gutierrez-Gonzalez ${ }^{1}$, F. Herrera ${ }^{2}$, M.T. Fernandez Mestre ${ }^{2}$ on behalf of GRUVES (Grupo Venezolano para el Estudio de las Espondiloartritis). ${ }^{1}$ Centro Nacional de Enfermedades Reumaticas (Cner): ${ }^{2}$ Laboratorio de Fisiopatología, Instituto Venezolano de Investigaciones Científicas (IVIC), Caracas, Venezuela, Bolivarian Republic Of

Background: Psoriatic Arthritis (PsA) is not a regular systemic autoimmune disease, many expert define it with autoinflammatory disease, resulting in chronic inflammation of the synovium and consequent cartilage and bone erosion in approximately $10 \%$ of patients with skin psoriasis. It is important to identify novel genomic biomarkers associated with disease susceptibility but also able to detect early those patients with negative prognostic factors who may benefit from a more aggressive therapeutic approach. The over-expression of tumor necrosis factor (TNF)- $a$ is a central element in the pathogenesis of psoriasis and psoriatic arthritis (2). The levels of TNF-alpha are under genetic control. An "A" at position -308 in the TNFA promoter has been shown to be associated with increased level of TNF-alpha expression and "A" at position -238 with a diminished level of TNF- $\alpha$ expression $(3,4)$. Many authors consider that it is a disease only of Europeans descendants or Caucasians, however never be studied in mestizo population Objectives: We investigate the potential association between the TNFA-238 G/A, TNFA-308 G/A, IL10 -1082 G/A,-819C/T, -592C/A polymorphisms and the Psoriatic Arthritis susceptibility.

Methods: The study included 52 PsA patients diagnosed by CASPAR criteria and 52 controls. The polymorphism of TNFA-308 G/A (rs1800629), TNFA-238 G/A (rs361525), IL10 -1082 G/A (rs1800896), -819C/T (rs1800871) and -592C/A (rs1800872) were genotyped by single specific primers -polymerase chain reaction (SSP-PCR). All subjects were from an unrelated Venezuelan-Mestizo population with a history of ancestors, at least back to the third generation

Results: When comparing allele and genotype frequencies between the groups studied, no significant differences were observed for the TNFA-308 $\mathrm{G} / \mathrm{A}(\mathrm{rs} 1800629)$ and IL10 -1082 G/A (rs1800896), -819C/T (rs1800871) and $-592 \mathrm{C} / \mathrm{A}$ (rs1800872). However, our results showed a significant decrease in the frequency of the TNF-238A allele among PsA patients compared to healthy individuals ( $3.8 \%$ vs. $10.57 \%$, respectively, OR: $0.31,95 \% \mathrm{Cl}: 0.92-1.05$, p: 0.02$)$, suggesting that TNFA-238A allele may have a protective effect (Table 1).

Table 1. Genotypic and allelic frequencies of the 308 G / AG (rs1800629) and -238 G / A (rs36152! variants of the TNFA gene in patients with psoriatic arthritis and healthy controls

\begin{tabular}{|c|c|c|c|c|}
\hline $\begin{array}{c}\text { TNFA-308 G/A } \\
\text { (rs1800629) }\end{array}$ & $\begin{array}{c}\text { PsA } \\
n=73\end{array}$ & $\begin{array}{c}\text { Controls } \\
n=52\end{array}$ & $\begin{array}{c}\text { OR } \\
\text { (IC95\%) }\end{array}$ & $P$ \\
\hline \multicolumn{5}{|l|}{ Genotype } \\
\hline GG & $80,7 \%$ & $84,6 \%(44)$ & $0,76(0,27-2,12)$ & 0,302 \\
\hline GA & $19,3 \%$ & $15,4 \%(8)$ & $1,30(0,47-3,63)$ & 0.302 \\
\hline AA & $0 \%$ & $0 \%(0)$ & & \\
\hline \multicolumn{5}{|l|}{ Allele } \\
\hline G & $92,3 \%$ & $90,4 \%(94)$ & $1,27(0,48-3,37)$ & 0,311 \\
\hline A & $7,3 \%$ & $9,6 \%(10)$ & $0,78(0,29-2,07)$ & 0,311 \\
\hline $\begin{array}{c}\text { TNFA-238 G/A } \\
\text { (rs361525) }\end{array}$ & $\begin{array}{l}\text { PsA } \\
n=73\end{array}$ & $\begin{array}{c}\text { Controls } \\
n=52\end{array}$ & $\begin{array}{c}\text { OR } \\
\text { (IC95\%) }\end{array}$ & $P$ \\
\hline \multicolumn{5}{|l|}{ Genotype } \\
\hline GG & $92,3 \%$ & $78,8 \%(41)$ & $3,22(0,95-10,8)$ & $* 0,025$ \\
\hline GA & $7,7 \%$ & $21,2 \%(11)$ & $0,31(0,09-1,05)$ & ${ }^{*} 0,025$ \\
\hline AA & 0 & 0 & & \\
\hline \multicolumn{5}{|l|}{ Allele } \\
\hline G & $96,2 \%$ & $89,4 \%(93)$ & $2,95(0,91-9,61)$ & ${ }^{*} 0,0306$ \\
\hline A & $3,8 \%$ & $10,6 \%(11)$ & $0,39(0,10-1,09)$ & ${ }^{*} 0,0306$ \\
\hline
\end{tabular}

NOTE: The values shown in parentheses represent the number of individuals carrying the genotype for the polymorphic site studied. The frequency is expressed as a percentage ( $p>0.05$ : not significant, $p<0.05$ : significant) psoriatic arthritis and healthy controls

Conclusions: This is the first genetic study carried out in Venezuelan mestizo patients with Psoriatic Arthritis to establish associations between genetic markers such as the polymorphisms of the promoter region of the TNFA and IL10 genes and the disease. In conclusion the TNFA-238 G/A polymorphism might play an important role in the development of Psoriatic Arthritis in Venezuelan mestizos and this association could not only clarify the different factors involved in a multifactorial disease, such as Psoriatic Arthritis, but also establish the relationship of these molecular markers with some clinical manifestations of the disease, which would allow in the future to determine the suitability or not to use some types of treatments, such as the use of anti-TNF therapy, for example. References:

[1] Morales-Zambrano, et al. Int J Clin Exp Med. 2014; 7(9): 2605-2614.

[2] Bowes J, Barton A. Discov Med. 2010;10 (52):177-83.

[3] Liu Y, Helms C, et al. PLoS Genet, 2008;4(3): 1000041.
Acknowledgements: To bioanalyst Esther Guzmán, Marion DiMuro, Elena Flores and Mercedes Flores from the Clinica El Ávila and the University Hospital of Caracas (HUC/UCV), as well as Eva Salazar, Omar Balbas y Fernando Hernandez (IVIC).

Disclosure of Interest: L. A. Gutierrez-Gonzalez Grant/research support from: IIR/PFIZER, F. Herrera Grant/research support from: Grant Award (Pfizer), M. Fernandez Mestre: None declared

DOI: 10.1136/annrheumdis-2017-eular.1490

\section{AB0005 CYTOKINE MRNA GENE EXPRESSION ASSOCIATED WITH SYSTEMIC LUPUS ERYTHEMATOSUS}

L. Miteva ${ }^{1}$, M. Ivanova Goycheva ${ }^{2}$, I. Manolova ${ }^{1}$, G. Vasilev $^{2}$, R. Stoilov ${ }^{2}$, S. Stanilova ${ }^{1}$. ${ }^{1}$ Department of Molecular Biology, Immunology and Medical Genetics, Medical Faculty, Trakia University, Stara Zagora; ${ }^{2}$ Clinic of Rheumatology, University Hospital "St. Iv. Rilski", Medical Faculty, Medical University, Sofia, Bulgaria

Background: Systemic lupus erythematosus (SLE) is a complex polygenic autoimmune disease, characterized by autoantibody production, inflammatory manifestation and imbalanced cytokine production. In accordance with the pivotal role of Th17 cell in autoimmunity and the altered Th17/Tregs balance in response to changes in the cytokine milieu we analyzed mRNA expression of several cytokines in peripheral blood of SLE patients. Quantification of mRNA expression in peripheral blood could be useful to assess the disease activity of SLE patients. Objectives: The aim of the present study was to investigate the gene expressions at mRNA level of proinflammatory (TNFA, IL18, IL12B); Th17-related (IL23A); immunosuppressive (TGFB1 and IL10) cytokines and Treg-specific transcription factor Foxp3 in peripheral blood of women with SLE.

Methods: Total RNA from peripheral blood was isolated from 28 female patients with SLE and 17 healthy women. Quantitative real-time polymerase chain reaction was performed for 7 genes of interests, using the TaqMan detection system. Relative quantitative evaluation of mRNAs was performed by the comparative $\Delta \Delta \mathrm{Ct}$ method and results are presented as $\mathrm{n}$-fold mean difference (RQ-relative quantity) of target genes relative to calibrator (healthy controls) after normalization to the reference gene-GAPDH. Disease activity in SLE was determined by SLEDAI and divided into three categories: mild (0-5), moderate (6-10) and high $(>10)$.

Results: The results reveal considerable overexpression of IL23A in SLE patients compared to healthy controls $(R Q=5.347 ; p<0.001)$. According to the level of disease activity we found the highest elevation of IL23A in patients with SLEDAl $>10(R Q=8.54 ; p<0.001)$ compared to the controls. In inactive to mild (SLEDAI 0-5) and moderate SLE (SLEDAI 6-10), IL23A was also upregulated in approximately equal rate $(R Q=4.976 ; p<0.001$ and $R Q=4.64 ; p<0.001)$. In addition, immunosuppressive cytokines IL10 and TGFB1 mRNA were elevated significantly in SLE patients than in controls $(R Q=1.79 ; p=0.0077$ and $R Q=1.78$; $\mathrm{p}=0.02$, respectively). We also found that the expression of proinflammatory TNFA and IL12B were significantly downregulated approximately 2 -fold. The mRNA level of Foxp3 was downregulated only for patients with SLEDAI>10. A significant good correlation between IL18 and the SLEDAI score was found $(r=0.5548$; $\mathrm{p}=0.002)$. Higher IL18 expression was observed among patients with worsened SLE compared to those with mild $(R Q=1.656 ; p=0.008)$ and moderate $(R Q=1.474$; $\mathrm{p}=0.034$ ) disease activity. We further demonstrated positively correlation between the expression levels of IL23A and TGFB1 ( $r=0.7276 ; p<0.001)$ among SLE patients.

Conclusions: These results suggest that upregulation of IL23 and TGFB1 in addition to downregulated Foxp3 expression may contribute to skewing towards Th17 profile in SLE pathogenesis and this was the most markedly manifested at the highest level of disease activity. Our results support indirectly the idea for restoring Th17/Treg balance as a therapeutic target in SLE.

Disclosure of Interest: None declared

DOI: 10.1136/annrheumdis-2017-eular.2299

\section{AB0006 MICRORNA-499 IN BEHCET DISEASE AND POSSIBLE ASSOCIATION WITH DISEASE ACTIVITY}

M. Eissa ${ }^{1}$, M. Al Kaffas ${ }^{2}$, S. Abdel Wahab ${ }^{2}$, L. Rashed ${ }^{2} .{ }^{1}$ Department of rheumatology and rehabilitation; ${ }^{2}$ Medical Biochemistry, Cairo University, Cairo, Egypt

Background: Behçet Disease (BD) is a relapsing inflammatory autoimmune disease. Although the etiology of BD is not yet known, genetic predisposition and immune dysregulation are thought to be critical factors in the pathogenesis of the disease. MicroRNAs (miRNAs) are small RNA fragments that can regulate the gene expression. miRNAs play a critical role in the pathogenesis of autoimmune or auto inflammatory diseases. Sinngle nucleotide polymorphisms (SNPs) may change the property of miRNAs through altering miRNA expression and/or maturation.

Objectives: The aim of this work was to detect miRNA-499 (rs3746444) genotyping and relative expression in BD patients in order to find out the possible association between them and disease activity and severity.

Methods: Relative expression of miRNA-499 was measured by Real-Time PCR and miRNA-499 gene (rs 3746444) genotyping was performed by TaqMan SNP 